\title{
Role of PET/CT in initial evaluation of lymphoma patients
}

\author{
Manar Mohamed Naguib*, Samer Malak Botros, Amir Louis Louka and Rasha S. Hussein
}

\begin{abstract}
Background: Accurate radiologic assessment of treatment response in lymphoma patients is important to evaluate the effectiveness of treatment and consequently predict the relapse; the value of PET/CT for post-treatment prognosis prediction has been recently investigated. The aim of this study is to highlight the prognostic value of PET-CT metabolic volumetric parameters in the evaluation of lymphoma patients.

Results: Among the included 40 patients, post-treatment SUV, MTV, and TLG were significantly lower in a responsive group than the non-responsive group. \% changes of all quantitative PET/CT parameters were significantly higher in the responsive group than the non-responsive group.

Conclusions: This study suggests that pre-treatment PET/CT quantitative measures (except baseline SUVmax) are not conclusive in the prediction of patient response to treatment; however, the $\triangle S U V \max , \triangle M T V$, and $\triangle T L G \%$ from the baseline to the end of therapy could be used in predicting patient response to treatment, determining patient prognosis, and suggesting the relapse.
\end{abstract}

Keywords: Hodgkin lymphoma, Non-Hodgkin lymphoma, 18F FDG PET/CT, PERCIST, CMR, PMR, SMD, PMD

\section{Background}

Lymphomas are a heterogeneous group of malignancies that can be divided into two main groups: Hodgkin lymphoma (HL) and Non-Hodgkin lymphoma (NHL) [1]. Once the diagnosis of lymphoma is made by biopsy, determination of disease extent, i.e., staging is important for appropriate treatment planning and determining the prognosis [2].

Although major progress is made in the treatment of lymphoma patients, many still fail to achieve response and subsequently they relapse $[3,4]$. These patients are not easily identified by the existing pre-treatment prognostic indexes such as the IPI (international prognostic index), FLIPI (prognostic score for follicular lymphoma), or by CT-based response assessment. Therefore, there is an urgent need for new prognostic and predictive

\footnotetext{
*Correspondence: manarmohamed.mm9@gmail.com

Radiology Department, Faculty of Medicine, Ain Shams University, 54

Mostafa Basha Street, El-Zayton, Cairo, Egypt
}

markers which permit early identification of high-risk patient categories [5-8].

FDG-PET/CT has been recognized as a standard imaging modality in FDG-avid lymphomas [9, 10]. However, there are still several suggestions for response evaluation methods using FDG PET, in terms of target lesions and quantitative indexes. In PERCIST system [11-13], it is recommended to measure the standardized uptake value (SUV) of a single representative lesion, whereas RECIST1.1 recommends measuring tumor diameters of maximum five lesions with no more than two lesions of a single organ. Thus, methods for treatment response evaluation using quantitative indexes of FDG PET need more refinement and validation [14-16].

The aim of this study is to highlight the diagnostic and prognostic values of baseline and post-treatment PETCT quantitative metabolic volumetric parameters in the evaluation of lymphoma patients. 


\section{Methods}

\section{Patients}

This prospective study was conducted between December 2019 and October 2020. Forty patients with biopsyproven lymphoma (including both $\mathrm{HL}$ and NHL) performed baseline (pre-treatment) and post-therapy (6-8 weeks after the end of chemotherapy) PET/CT imaging studies. The study was performed after approval of the Ethical Committee of Scientific Research, Faculty of Medicine. Consent was taken from all patients. Any patient presented with lymphoma who did not receive any therapy yet was included. Both sexes were included with no age predilection. We excluded any patient with atopic disorders, bad general condition, or renal impairment. Patients who underwent any surgical intervention or received radiotherapy were also excluded.

\section{Patient preparation}

Procedure time was at diagnosis and 6-8 weeks after the end of the chemotherapy. Patients fasted for $6 \mathrm{~h}$ before the examination but with good hydration. Exercise was avoided minimum for $2 \mathrm{~h}$ before the scan. Pre-scanning blood glucose level estimation (below $200 \mathrm{mg} / \mathrm{dl}$ ) was done, and insertion of an intravenous cannula was performed.

\section{Technique of 18F-FDG PET/CT scan}

A radioactive tracer (18F-FDG) was injected intravenously with a dose of $0.06-0.08 \mathrm{mCi} / \mathrm{kg}$. All patients were kept in a warm room, asked to rest and void just before imaging. Scanning by hybrid PET/CT scanner (GE Discovery IQ 5 rings) was performed 60 min after injection. The patient was positioned supine on the table. Initial single-phase contrast-enhanced helical CT (optima 540 16 slice) was performed following an injection of $125 \mathrm{ml}$ of a low osmolarity iodinated contrast medium (Optiray 350 ) at a rate of $4 \mathrm{ml} / \mathrm{s}$ by using power injector (Discovery IQ, GE Medical System, USA). CT scanning (from the head to mid-thigh) was obtained using $110 \mathrm{~mA}, 110 \mathrm{kV}$, $0.5 \mathrm{~s}$ tube rotation time, and $3.3 \mathrm{~mm}$ section thickness. After CT scanning, PET scan covering the same field of view was obtained immediately. Six- to seven-bed positions were planned in three-dimensional acquisition mode for scanning the entire patient with 3-5 min acquisition at each position. Images were transferred to the workstation (Advantage Window 4.7) to be reconstructed and displayed in axial, coronal, and sagittal planes. Fused images were obtained.

\section{Image analysis}

Images were analyzed by two experienced radiologists. Analysis of CT images was done by visual inspection for the selection of the target lesions. Analysis of PET/CT images was done; the target lesions are of highest SUVmax with maximum 5 lesions per patient; quantitative calculation of FDG uptake was corrected to lean body mass. We measured SUL peak to perform PERCIST 1.0 criteria as the gold standard in PET/CT interpretation. Calculation of initial and post-treatment SUVmax, MTV, and TLG with measuring $\%$ changes $(\triangle \mathrm{SUV}, \triangle \mathrm{TLG}$, and $\Delta \mathrm{MTV})$ per lesion was performed. Patients are categorized into four groups according to treatment response as PMR (partial metabolic response), i.e., decrease in greater than or equal to $30 \%$ of SUL peak, CMR (complete metabolic response), i.e., no uptake, SMD (stable metabolic disease), i.e., decrease or increase in SUL peak of less than 30\%, and PMD (progressive metabolic disease), i.e., increase in SUL peak of greater than $30 \%$.

\section{Statistical analysis}

Statistical analysis was performed using MedCalc statistical software for Windows (MedCalc Software, Mariakerke, Belgium). Data for continuous variables were expressed as either median, interquartile range or mean \pm standard deviation and as both number and percentage for categorical data. Mann-Whitney $U$ test was used to evaluate the differences in continuous variables between the responsive and non-responsive groups. The Wilcoxon test for paired samples was used to compare the pre- and post-treatment parameters in all groups. Comparisons of all parameters between the PMR, CMR, SMD and PMD groups were performed using the Kruskal-Wallis test, and Conover post hoc test was used for pairwise comparisons of the different groups. Receiver operator characteristic (ROC) curve analysis was performed to determine optimum thresholds and the diagnostic accuracy of the various variables in distinguishing the different groups. The diagnostic accuracy of all variables was evaluated in terms of sensitivity, specificity, positive predictive value (PPV), negative predictive value (NPV), and area under the ROC curve (AUC). For all tests, all $P$ values were two-tailed and a $P$ value $<0.05$ was considered significant.

\section{Results}

The study included 40 patients (20 males and 20 females). Their ages ranged from 16 to 65 years. Histopathological analysis revealed that $24(60 \%)$ patients had classical HL, while 16 (40\%) patients had NHL.

A total of 103 lesions were assessed in 40 patients. Response to treatment was evaluated on a per lesion basis according to PERCIST 1.0 criteria and based upon findings; 61 (59.2\%) lesions showed CMR, 28 (27.2\%) lesions showed PMR, 8 (7.8\%) lesions showed SMD, and 6 (5.8\%) lesions showed PMD. Lesions with an outcome of progressive or stable disease were designated as 
non-responsive lesions, while those of partial complete response were grouped together as responsive lesions.

Comparison of the pre- and post-treatment SUVmax, MTV, and TLG in the responsive and non-responsive lesions groups revealed that all post-treatment values were significantly lower than the pre-treatment values $(P<0.0001)$ in the responsive group, while no significant difference existed between baseline and post-treatment SUVmax $(P=0.18)$, MTV $(P=0.68)$, and TLG $(P=0.91)$ values in the non-responsive group.

When responsive and non-responsive groups were compared, post-treatment SUVmax, post-treatment MTV, and post-treatment TLG were significantly lower in the responsive group than the non-responsive group $(P<0.0001, P<0.0001$, and $P<0.0001$, respectively). Percent changes of all quantitative $\mathrm{PET} / \mathrm{CT}$ parameters were significantly higher in the responsive group than the non-responsive group $(P<0.0001)$. No significant difference was found between the baseline MTV and TLG of both groups ( $P=0.11$ and $P=0.90$, respectively) (Table 1 ; Figs. 1, 2).

In an effort to determine whether quantitative PET/ CT parameters can differentiate between the individual groups, namely the CMR, PMR, SMD and PMD groups, comparisons of the various variables between the four groups were also performed (Table 2). All post-treatment quantitative PET/CT parameters were significantly lower than pre-treatment values in PMR, CMR, and SMD groups and significantly higher than pre-treatment values in the PMD group. There were statistically significant differences in $\triangle \mathrm{SUV}, \triangle \mathrm{MTV}$, and $\triangle \mathrm{TLG}$ between all four groups (Figs. 3, 4).

\section{Discussion}

18F-FDG PET/CT is currently a standard imaging technology for diagnosis, staging, and prediction of prognosis in patients with HL or NHL. It has been extensively utilized in the managing of malignant lymphoma patients,

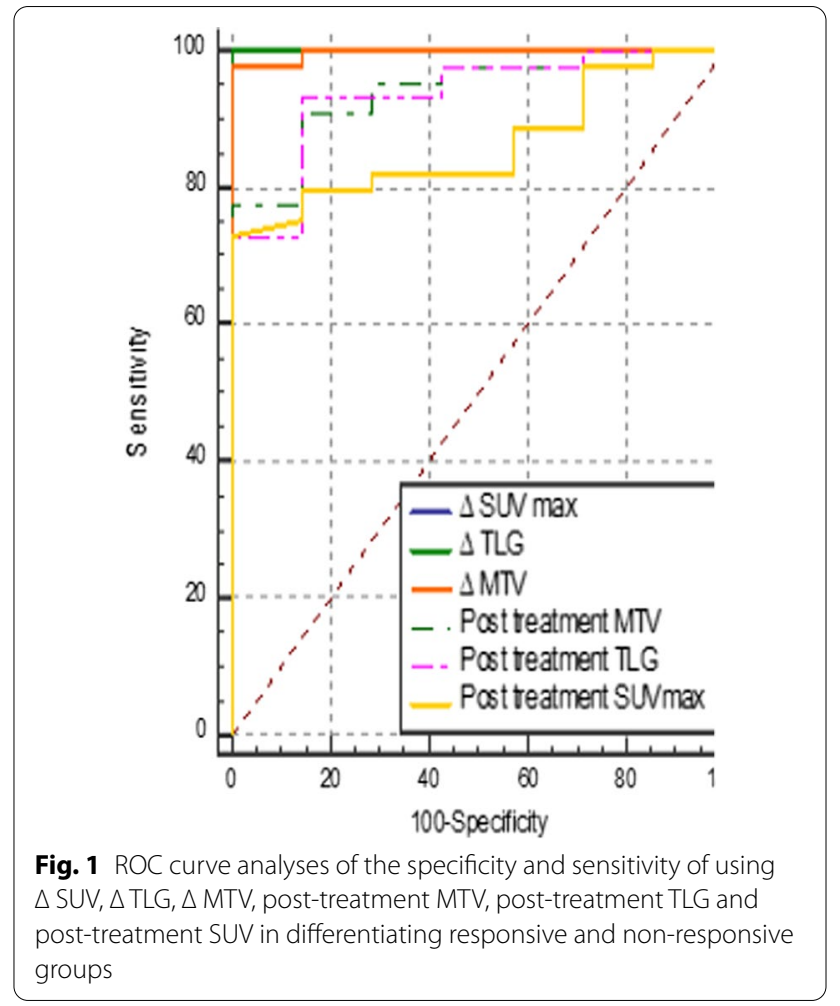

and there is increasing evidence of the prognostic significance of PET/CT parameters [17].

In analyzing FDG PET, SUVmax is the most widely used index for various purposes relatively because of the suitability and great reproducibility of measurement; it reveals the metabolic activity of the most aggressive tumor cell. Recently, researchers have been minded by metabolic tumor volume (MTV) which is a measurement of tumor volume with a high metabolism, and total lesion glycolysis (TLG), which is the product of mean SUV and MTV. MTV and TLG are volume-based indexes that reflect tumor burden, and they are expected to be effective in prognosis prediction and response evaluation $[1$,

Table 1 The sensitivities, specificities, PPV, NPV, accuracies, AUC, and P values of the optimum threshold values of the quantitative PET/CT parameters for differentiation of responsive from the non-responsive group

\begin{tabular}{|c|c|c|c|c|c|c|c|}
\hline Parameter & $\begin{array}{l}\text { Optimum threshold } \\
\text { value }\end{array}$ & Sensitivity & Specificity & PPV & NPV & AUC & $P$ value \\
\hline Baseline SUV & $>4.55$ & $83.15 \%$ & $85.71 \%$ & $97.4 \%$ & $44.4 \%$ & 0.798 & 0.0001 \\
\hline Post-treatment SUV & $\leq 1.4$ & $73 \%$ & $100 \%$ & $100 \%$ & $36.8 \%$ & 0.864 & $<0.0001$ \\
\hline$\triangle S U V$ & $>28$ & $100 \%$ & $100 \%$ & $100 \%$ & $100 \%$ & 1.000 & $<0.0001$ \\
\hline Post-treatment MTV & $\leq 1$ & $77.5 \%$ & $100 \%$ & $100 \%$ & $41.2 \%$ & 0.939 & $<0.0001$ \\
\hline$\triangle \mathrm{MTV}$ & $>42.9$ & $97.75 \%$ & $100 \%$ & $100 \%$ & $87.5 \%$ & 0.997 & $<0.0001$ \\
\hline Post-treatment TLG & $\leq 15.3$ & $93.26 \%$ & $85.71 \%$ & $97.6 \%$ & $66.7 \%$ & 0.936 & $<0.0001$ \\
\hline$\Delta \mathrm{TLG}$ & $>55$ & $100 \%$ & $100 \%$ & $100 \%$ & $100 \%$ & 1.000 & $<0.0001$ \\
\hline
\end{tabular}




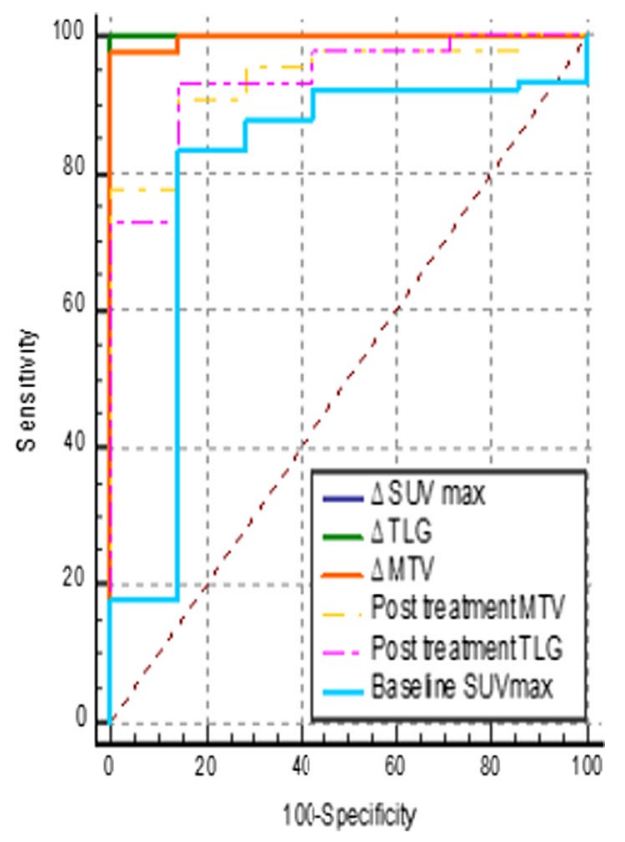

Fig. $2 R O C$ curve analyses of the specificity and sensitivity of using $\triangle S U V, \triangle T L G, \triangle M T V$, post-treatment MTV, TLG and baseline SUV in differentiating responsive and non-responsive groups

18, 19]. In our study, a total of 103 lesions were assessed in 40 patients, and quantitative indexes at baseline and the end of treatment (6-8 weeks after end of chemotherapy) are measured. We compare all baseline and posttherapy PET/CT parameters between the four groups, namely PMR (partial metabolic response) and CMR (complete metabolic response), which are collectively known as responsive lesions, SMD (stable metabolic disease) and PMD (progressive metabolic disease): grouped together as non-responsive lesions. We found that baseline SUV of the CMR group was significantly higher than that of SMD group, yet it is lower than that of PMR group. The pre-treatment SUV of PMR group was significantly higher than the SMD and PMD groups. The posttreatment SUV of the CMR group was significantly lower than that of all other groups. The post-treatment SUV of PMD group was significantly higher than all other groups (Fig. 5).

Our study results matched the study carried out by Huang et al. [20] in which response to treatment was assessed in patients after the completion of six to eight cycles of treatment. The complete remission rate [complete response $(\mathrm{CR})+$ unconfirmed complete response $(\mathrm{Cru})]$ and overall response rate after therapy were significantly higher in the low SUVmax group (pre-treatment SUVmax $\leq 9.0$ ) than in the high SUVmax group.

There are several subtypes of aggressive lymphoma. These include AID-associated lymphoma, Burkitt lymphoma, CNS lymphoma, diffuse large B-cell lymphoma (DLBCL), mantle cell lymphoma, and peripheral T-cell lymphoma. Another significant impact of baseline SUVmax is the prediction of the presence of more aggressive histological components and suggestion of foci of aggressive transformation which has potential diagnostic and therapeutic implications. Ngeow et al. [21] observe that in a patient with indolent lymphoma, sites with SUV of $>10$ suggest the possibility of transformation or the possibility of the presence of an aggressive component in addition to what is suggested by the histology. In our study, we noticed that patient with more aggressive histological types as T-cell lymphoma or DLBCL has the highest baseline SUVmax reaching about 26 and 17, respectively, yet the prediction of treatment response is multifactorial that is not only depend on histological type of the tumor.

Recent studies suggest that MTV and TLG are more inclusive parameters that better represent the whole metabolic tumor burden than SUVmax, but with a limitation point that they require accurate standardization of tumor segmentation. These studies suggest the usefulness of MTV and TLG for expectation of treatment response and prediction of prognosis [17]. However, in our study, baseline MTV and TLG did not surpass baseline SUVmax in terms of initial (baseline) response evaluation. No significant difference was found in our study between the baseline MTV and TLG in both responsive and nonresponsive groups. Although we notice that the range of baseline MTV and TLG is low in responsive than nonresponsive groups, it is clinically insignificant and their statistical analysis is insignificant (as their $P$ values 0.11 and 0.9, respectively); the increase in baseline MTV and TLG is noticeable mainly in non-responder groups; however, it is statistically not accurate predicting of treatment response (Fig. 6).

In contrast to our finding, Albano et al. [22] conducted a prospective study that included 123 elderly patients initially diagnosed with HL. Comparison of baseline metabolic PET/CT quantitative parameters between no complete and complete response groups after first-line treatment was made. The baseline L-L SUV R (lesion-toliver ratio), baseline L-BP SUV R (lesion-to-blood pool ratio), baseline MTV, and baseline TLG were significantly lower in the complete metabolic response group than in the no-complete response group ( $P$ values of $0.032,0.042$, 0.004 , and 0.005 , respectively).

Akhtari et al. [23] assumed that three-dimensional measurement of tumor burden measured on baseline PET/CT such as MTV and TLG might more precisely risk-stratify the patients. They evaluated 267 patients with a median follow-up of 4.96 years, of which 27 patients had relapse or refractory disease and 12 died. 
Table 2 Comparison of quantitative PET/CT parameters between all groups

\begin{tabular}{|c|c|c|c|c|c|}
\hline & Partial response & Complete response & Stable disease & Progressive disease & $P$ value \\
\hline \multicolumn{6}{|c|}{ Baseline SUV } \\
\hline Median & 12.6 & 9.7 & 3.4 & 2.7 & \multirow[t]{3}{*}{$P=0.0004 A$} \\
\hline IQR & $9-15.8$ & $5.6-14$ & $2.8-4.2$ & $1.8-16.4$ & \\
\hline Range & $3.1-29$ & $1.3-25$ & $2.5-4.6$ & $1.8-16.4$ & \\
\hline \multicolumn{6}{|c|}{ Post-treatment SUV } \\
\hline Median & 3.3 & 0 & 3 & 6.3 & \multirow[t]{3}{*}{$P<0.000001 \mathrm{~B}$} \\
\hline IQR & $2-4.8$ & $0-0$ & $2.4-3.7$ & $2.7-22.1$ & \\
\hline Range & $1.18-8.2$ & $0-0$ & $1.8-4.5$ & $2.7-22.1$ & \\
\hline \multicolumn{6}{|l|}{$\triangle S U V(\%)$} \\
\hline Median & 74.5 & 100 & 11.9 & -53.4 & \multirow[t]{3}{*}{$P<0.000001$} \\
\hline IQR & $65.3-80.8$ & $100-100$ & $1.1-24.8$ & -137.7 to 35.1 & \\
\hline Range & $41.9-87.3$ & $100-100$ & $0-28$ & -137.7 to -35.1 & \\
\hline \multicolumn{6}{|c|}{ Baseline MTV } \\
\hline Median & 29.8 & 5.8 & 42.5 & 31.2 & \multirow[t]{3}{*}{$P=0.011 C$} \\
\hline IQR & $4.8-78$ & $2.8-23.1$ & $6.9-264.5$ & $2.9-39$ & \\
\hline Range & $1.13-215$ & $1.13-182$ & $2.8-455$ & $2.9-39$ & \\
\hline \multicolumn{6}{|c|}{ Post-treatment MTV } \\
\hline Median & 2.6 & 0 & 32.7 & 38.4 & \multirow[t]{3}{*}{$P<0.000001 D$} \\
\hline IQR & $1-6$ & $0-0$ & $6.3-238.2$ & $5.1-47.7$ & \\
\hline Range & $0.5-92$ & $0-0$ & $1.6-422$ & $5.1-47.7$ & \\
\hline \multicolumn{6}{|l|}{ MMTV (\%) } \\
\hline Median & 75.5 & 100 & 16.95 & -23.1 & \multirow[t]{3}{*}{$P<0.000001$} \\
\hline IQR & $55.8-94.4$ & $100-100$ & $3.7-34.8$ & -76.2 to -22.4 & \\
\hline Range & 39.9-99.4 & $100-100$ & $0-42.9$ & -76.2 to -22.4 & \\
\hline \multicolumn{6}{|c|}{ Baseline TLG } \\
\hline Median & 250 & 25 & 100 & 49.1 & \multirow[t]{3}{*}{$P=0.037 \mathrm{E}$} \\
\hline IQR & $24.7-1081$ & $11.3-173.7$ & $13.5-685.5$ & $4.7-306.5$ & \\
\hline Range & $6.8-2038$ & $2.6-1804$ & $4-1194$ & $4.7-306.5$ & \\
\hline \multicolumn{6}{|c|}{ Post-treatment TLG } \\
\hline Median & 6.5 & 0 & 61 & 60.4 & \multirow[t]{3}{*}{$P<0.000001 \mathrm{~F}$} \\
\hline IQR & $2.3-15.3$ & $0-0$ & $12.4-564.5$ & $17.1-528.2$ & \\
\hline Range & $0.8-117.6$ & $0-0$ & $1.8-1030$ & $17.1-528.2$ & \\
\hline \multicolumn{6}{|l|}{$\Delta T L G(\%)$} \\
\hline Median & 91.4 & 100 & 28.9 & -65.5 & \multirow[t]{3}{*}{$P<0.000001$} \\
\hline IQR & $85.6-99.2$ & $100-100$ & $6.9-49.6$ & -263.8 to -23 & \\
\hline Range & 71.2-99.9 & $100-100$ & $0-55$ & 263.8 to -23 & \\
\hline
\end{tabular}

They stated that baseline MTV and TLG interrelated significantly with freedom of progression (FFP); patients with TMTV 268 and TLG' 1703 had shorter FFP times and were to harbor bulky disease and staged as IIB-advanced disease.

On the contrary, Mettler et al. [24] in a retrospective study including 310 patients with baseline PET/CT scans available stated that baseline TMTV unsuccessfully predicted PFS and OS in patients diagnosed with advanced-stage Hodgkin lymphoma.
The utility of PET/CT in assessing response after the end of treatment has been confirmed in several studies. End-of-treatment FDG PET/CT is used to evaluate the efficacy of treatment, monitoring of residual tumor and predicting relapse $[25,26]$. The percentage of $\triangle S U V \max$ (between baseline and end of therapy) is a semiquantitative method with excellent inter-observer agreement and improved prognostic value of E-PET (end-of-therapy PET) [21]. Itti et al. [27] reported that $\Delta$ SUVmax\% $>72.9 \%$ is an important predictor of 


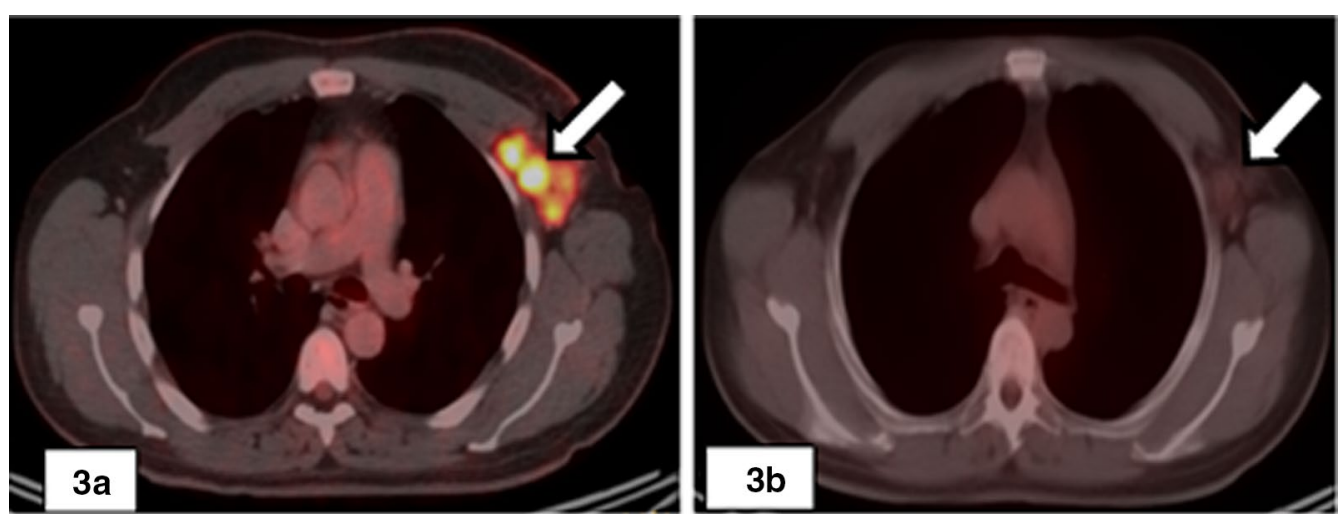

Fig. 3 36-year-old male patient, pathologically proved case of HL: initial PET/CT shows (a) FDG-avid amalgamated left axillary nodes (exhibiting: SUVmax of 8.87, MTV of $32.3 \mathrm{~cm}^{3}$, and TLG of $170 \mathrm{gm} / \mathrm{ml}$ ), post-treatment PET/CT showed (b) CMR (exhibiting: SUVmax of 2, MTV of $11.8 \mathrm{~cm}^{3}$, and TLG of $12.7 \mathrm{gm} / \mathrm{ml}$ )
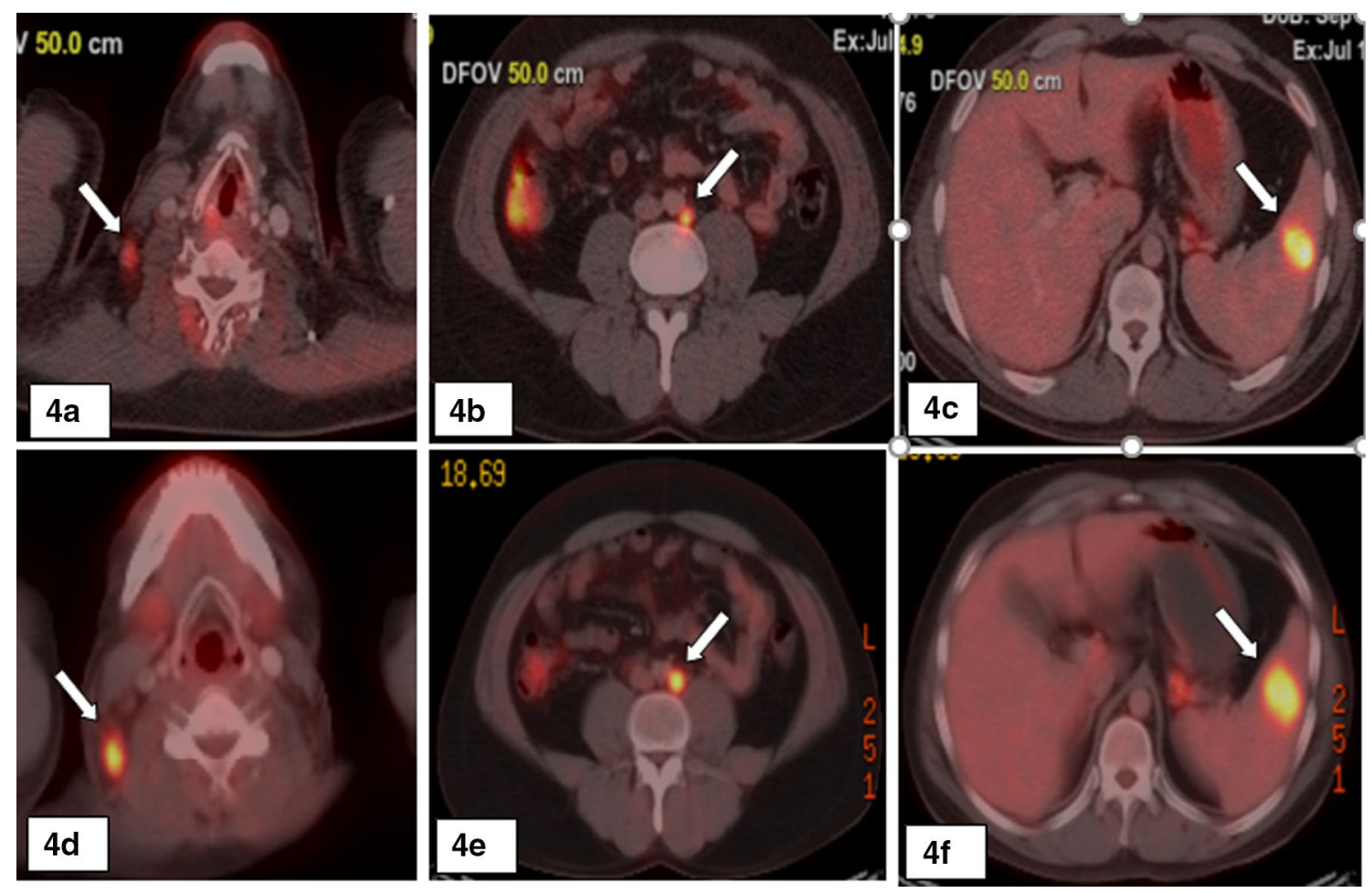

Fig. 4 35-year-old male patient, pathologically proved case of HL, initial PET/CT shows FDG-avid a right posterior triangle (exhibiting: SUVmax of 4.6, MTV of $2 \mathrm{~cm}^{3}$, and TLG of $18 \mathrm{gm} / \mathrm{ml}$ ), $\mathbf{b}$ left paraaortic lymph nodes (exhibiting: SUVmax of 4.5, MTV of $5.5 \mathrm{~cm}^{3}$, and TLG of $25 \mathrm{gm} / \mathrm{ml}$ ) and c extra-nodal splenic focal lesion (exhibiting: SUVmax of 6.9, MTV of $16 \mathrm{~cm}^{3}$, and TLG of $25 \mathrm{gm} / \mathrm{ml}$ ), post-treatment PET/CT (after 6 weeks) shows PMD as following: $\mathbf{d}$ right posterior triangle lymph node (exhibiting: SUVmax of 8, MTV of $6 \mathrm{~cm}^{3}$, and TLG of $25 \mathrm{gm} / \mathrm{m}$ ), e left paraaortic lymph node (exhibiting: SUVmax of 7.8, MTV of $14.9 \mathrm{~cm}^{3}$, and TLG of $33 \mathrm{gm} / \mathrm{ml}$ ), $\mathbf{f}$ splenic focal lesion (exhibiting: SUVmax of 8.5, MTV of $29 \mathrm{~cm}^{3}$, and TLG of 160 $\mathrm{gm} / \mathrm{ml})$

progression-free survival (PFS) at the end of treatment in DLBCL patients.

In our study, we noticed that $\triangle$ SUV and $\triangle$ TLG followed by $\triangle \mathrm{MTV}$, post-treatment MTV, post-treatment
TLG, and post-treatment SUV (in descending order) had the largest area under the receiver operating characteristic (ROC) curve which arranges PET/CT variable according to their diagnostic performance (sensitivity 


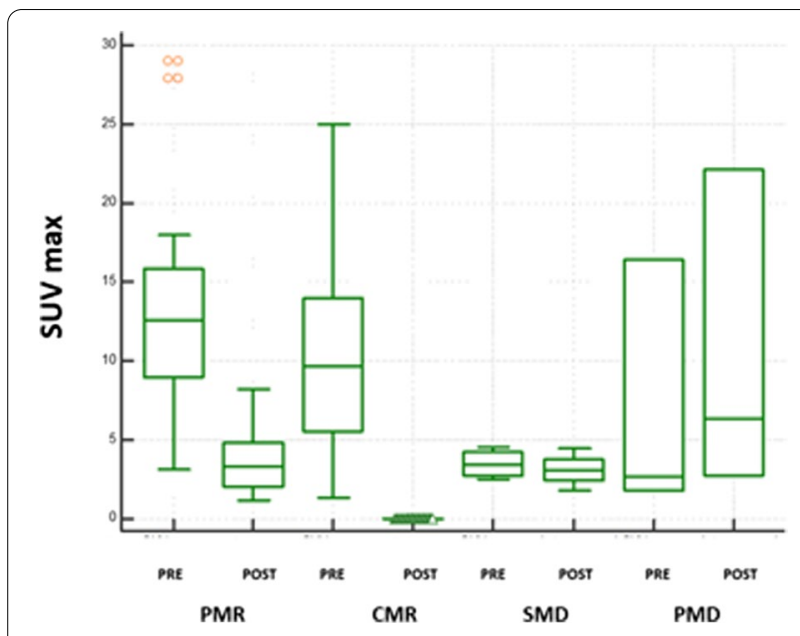

Fig. 5 Box-and-whisker plots of pre-treatment and post-treatment SUV in PMR, CMR, SMD, and PMD groups

and specificity) in distinguishing the responsive from the non-responsive group. All post-treatment quantitative $\mathrm{PET} / \mathrm{CT}$ parameters were significantly lower than pre-treatment values in PMR, CMR, and SMD groups and significantly higher than pre-treatment values in the PMD group.

Our study results are also matched with other studies which include baseline and post-treatment quantitative PET/CT parameters. In a study done by Zhou et al. [28] with 43 patients investigated, 28 patients underwent both baseline and end-of-treatment PET/CT scan; these patients were also evaluated for 1 and 2 years to estimate progression-free survival (PFS) and revealed that the $\triangle \mathrm{SUVmax} \%$ between baseline and end-of-therapy
PET was significantly different between the progression $(n=14)$ and progression-free groups $(n=14)(41.70 \%$ vs. $82.34 \%)$. When using $\Delta \mathrm{SUVmax} \%$ as a predictor of progression, patients with lower $\Delta$ SUVmax\% $(<66.95 \%)$ had low PFS compared with those with higher $\triangle S U V \max \%$ (>66.95\%). In our study, we suggested that $\triangle \mathrm{SUVmax}$ of CMR group was significantly higher than that of all other groups and the $\triangle$ SUVmax of the PMR group was significantly higher than that of SMD group. The $\triangle$ SUVmax of SMD group was also significantly higher than that of PMD groups. Also, $\triangle$ SUVmax of the PMD group was significantly lower than all other groups.

In Kim et al. study [29] of 57 patients, two target lesion sets were defined in each patient for analysis: (target A) a single hottest lesion and (target B) a maximum of five hottest lesions (where quantitative PET indexes of all lesions were summed into a single value). Quantitative indexes at initial and end-of-treatment (EOT) PET images were measured, and their percent differences $(\% \Delta)$ were calculated, which revealed that baseline SUVmax, baseline MTV, and all end-of-therapy PET parameters were significant prognostic factors with both targets $A$ and $B$, whereas TLG was not. Among them, baseline SUVmax presented the most significant ratio. In our study, we found that $\triangle M T V$ of CMR group was significantly higher than $\triangle \mathrm{MTV}$ of all other groups and the $\triangle M T V$ of the PMR group was significantly higher than that of SMD group. The $\triangle \mathrm{MTV}$ of SMD group was also significantly higher than the PMD groups. Also, $\triangle \mathrm{MTV}$ of the PMD group was significantly lower than that of all other groups.

On the contrary, we suggested that $\Delta$ TLG may play a future role in predicting the patient response as we found
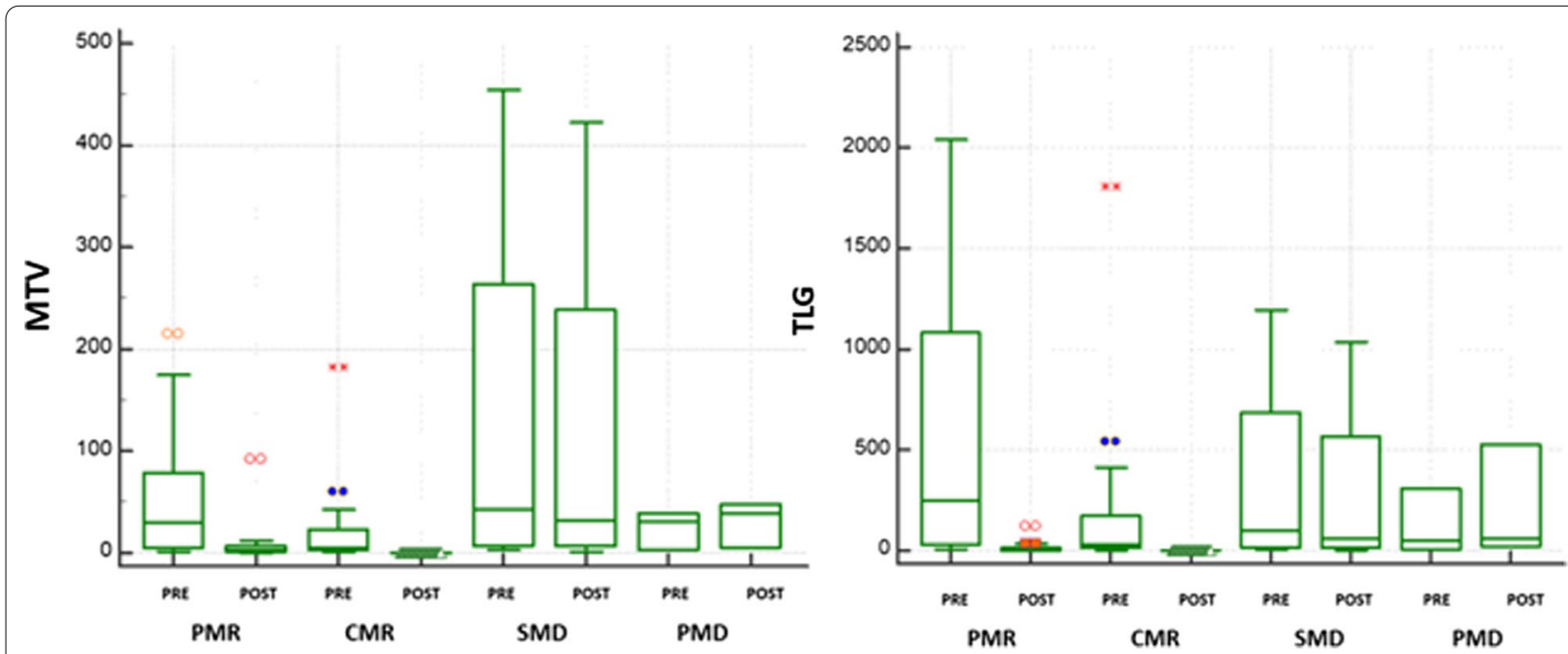

Fig. 6 Box-and-whisker plots of pre-treatment and post-treatment MTV and TLG in PMR, CMR, SMD, and PMD groups 
that $\triangle$ TLG of CMR group was significantly higher than that of all other groups and the $\triangle$ TLG of the PMR group was significantly higher than that of SMD group. The $\triangle T L G$ of SMD group was also significantly higher than that of PMD groups. Also, $\triangle$ TLG of the PMD group was significantly lower than all other groups.

Regarding the post-treatment quantitative parameters, we noticed that post-treatment SUV and post-treatment MTV of the CMR group were significantly lower than those of all other groups. Post-treatment SUV and posttreatment MTV of PMD group were significantly higher than those of all other groups. The post-treatment TLG of the CMR group was significantly lower than all other groups, while post-treatment TLG of the PMR group was significantly lower than SMD and PMD groups.

These results mean that from baseline PET/CT parameters only baseline SUVmax had a significant prognostic value in the evaluation of treatment response. $\triangle S U V m a x$, $\triangle \mathrm{MTV}$, and $\triangle \mathrm{TLG} \%$ from baseline to post-therapy $\mathrm{PET} / \mathrm{CT}$ seem to have a role in the prediction of patient prognosis.

There are few limitations of this study. First, the sample size is small with single-center experience due to the high cost of the technique. Second, adequate follow-up of patients was not achieved to correlate our results with the patients' progression-free survival or overall survival; multicenter study and research group cooperation using a large number of lymphoma patients may be needed to obtain more accurate results.

\section{Conclusions}

This study suggests that pre-treatment PET/CT quantitative measures (except baseline SUVmax) are not conclusive in the prediction of patient response to treatment; however, the $\triangle \mathrm{SUVmax}, \triangle \mathrm{MTV}$, and $\triangle \mathrm{TLG} \%$ from the baseline to the end of therapy could be used in prediction of patient response to treatment and determine patient prognosis. In view of these findings, future clinical trials are needed to determine the use of these parameters in various prognostic models (especially if correlated with PFS and OS), with the goal of achieving better risk stratification, patient prognosis, and treatment selection.

\begin{abstract}
Abbreviations
18F-FDG:: 18F-Fluorodeoxyglucose; PET/CT: Positron emission tomography/computed tomography; HL: Hodgkin lymphoma; NHL:: Non-Hodgkin lymphoma; PERCIST: PET response criteria in solid tumor; CMR: Complete metabolic response; PMR: Partial metabolic response; SMD:: Stable metabolic disease; PMD:: Progressive metabolic disease; SUV:: Standardized uptake value; MTV:: Metabolic tumor volume; TLG:: Total lesion glycolysis; IQR:: Interquartile range; PPV:: Positive predictive values; NPV:: Negative predictive values; AUC: Area under curve; PFS: Progression-free survival; OS: Overall survival.
\end{abstract}

Acknowledgements

Not applicable.

\section{Authors' contributions}

MM carried out the PET/CT studies, collected the data, and drafted the manuscript. SM, AL, and RS participated in the design of the study. MM performed the statistical analysis, and RS revised the manuscript. The authors read and approved the final manuscript.

\section{Funding}

This work has not received funding.

\section{Availability of data and materials}

The datasets used and/or analyzed during the current study are available from the corresponding author on reasonable request.

\section{Declarations}

\section{Ethics approval and consent to participate}

The study protocol was approved by the Research Ethics Committee (REC) of Ain Shams University, Faculty of Medicine, and reference number of approval: FWA 000017585 . All patients included in this study gave written informed to participate in this study.

\section{Consent for publication}

Written informed consent was obtained from all patients for publication of the data of this study.

\section{Competing interests}

The authors declare that they have no competing interests.

Received: 23 July 2021 Accepted: 22 November 2021

Published online: 01 December 2021

\section{References}

1. Buchpiguel CA (2011) Current status of PET/CT in the diagnosis and follow up of lymphomas. Rev Bras Hematol Hemoter 33(2):140-147

2. Kwee TC, Kwee RM, Nievelstein RJ (2008) Imaging in staging of malignant lymphoma: a systematic review. Blood 111:504-516

3. Burggraaff CN, De Jong A, Hoekstra OS et al (2019) Predictive value of interim positron emission tomography in diffuse large B-cell lymphoma: a systematic review and meta-analysis. Eur JNucl Med Mol Imaging 46:65-79

4. Cheson BD, Fisher RI, Barrington SF et al (2014) Recommendations for initial evaluation, staging, and response assessment of Hodgkin and nonHodgkin lymphoma: the Lugano classification. J Clin 32(27):3059-3068

5. Adams HJ, Nievelstein RA, Kwee TC (2015) Prognostic value of complete remission status at end-of-treatment FDG-PET in R-CHOP-treated diffuse large B-cell lymphoma: systematic review and meta-analysis. $\mathrm{Br} J$ Haematol 170(2):185-191

6. Adams HJ, Nievelstein RA, Kwee TC (2015) Prognostic value of interim FDG-PET in Hodgkin lymphoma: systematic review and meta-analysis. $\mathrm{Br}$ J Haematol 170(3):356-366

7. Gallamini A, Stelitano C, Calvi R et al (2004) Peripheral T-cell lymphoma unspecified (PTCL-U): a new prognostic model from a retrospective multicentric clinical study. Blood 103(7):2474-2479

8. Vaidya R, Witzig TE (2014) Prognostic factors for diffuse large B-cell lymphoma in the $R(X)$ CHOP era. Ann Oncol 25(11):2124-2133

9. Xie $M$, Zhai W, Cheng $S$ et al (2016) Predictive value of FDG PET /CT quantization parameters for progression-free survival in patients with diffuse large B-cell lymphoma. Hematology 21(2):99-105

10. Kanoun S, Tal I, Berriolo-Riedinger A et al (2018) Influence of software tool and methodological aspects of total metabolic tumor volume calculation on baseline [18F] FDG PET to predict survival in Hodgkin lymphoma. PLOS ONE 10:e0140830

11. Jh O, Lodge MA, Wahl RL (2016) Practical PERCIST: a simplified guide to PET response criteria in solid tumors 1.0. Radiology 280:576-584

12. Khong PL, Huang B, Lee EY et al (2014) Mid treatment FDG PET/CT scan for early response assessment of SMILE therapy in natural killer/T-cell lymphoma: a prospective study from a single center. J Nucl Med 55:911-916 
13. Metser U, Hussey D, Murphy G (2014) Impact of (18) F-FDG PET/CT on the staging and management of follicular lymphoma. Br J Radiol 87(1042):20140360

14. Persijn V, Meerten V, Gelderblom H et al (2010) RECIST revised implications for the radiologist: a review article on the modified RECIST guideline. Eur Radiol 20:1456-1467

15. Cheson BD, Kostakoglu L (2017) FDG-PET for early response assessment in lymphomas Part 2-diffuse large B-cell lymphoma: use of quantitative PET evaluation. Oncology (Williston Park) 31:71-76

16. Liao CC, Qin YY, Tan XH et al (2017) Predictive value of interim PET/CT visual interpretation in the prognosis of patients with aggressive nonHodgkin's lymphoma. Oncol Targets Ther 10:5727-5738

17. Freudenberg LS, Anotch G, Schütt $P$ et al (2004) FDG-PET/CT in restaging of patients with lymphoma. Eur J Nucl Med Mol Imaging 31:325-329

18. Chang Y, Fu X, Sun Z et al (2017) Utility of baseline, interim and endof-treatment (18) F-FDG PET/CT in extra-nodal natural killer/T-cell lymphoma patients treated with L-asparaginase/pegaspargase. Sci Rep 7:41057

19. Cheson BD, Kostakoglu L (2017) FDG-PET for early response assessment in lymphomas Part 1-Hodgkin lymphoma. Oncology (Williston Park) 31:45-49

20. Huang H, Xiao F, Han X et al (2016) Correlation of pretreatment 18F-FDG uptake with clinicopathological factors and prognosis in patients with newly diagnosed diffuse large B-cell lymphoma. Nucl Med Commun 37(7):689-698

21. Ngeow JY, Quek RH, Ng DC et al (2009) High SUV uptake on FDG-PET/ CT predicts for an aggressive B-cell lymphoma in a prospective study of primary FDG-PET/CT staging in lymphoma. Ann Oncol 20:1543-1547
22. Albano D, Mazzoletti A, Spallino M et al (2020) Prognostic role of baseline 18F-FDG PET/CT metabolic parameters in elderly HL: a two-center experience in 123 patients. Ann Hematol 99:1321-1330

23. Akhtari M, Milgrom SA, Pinnix CC et al (2018) Reclassifying patients with early-stage Hodgkin lymphoma based on functional radiographic markers at presentation. Blood 131(1):84-94

24. Mettler J, Müller H, Voltin CA et al (2019) Metabolic tumor volume for response prediction in advanced-stage Hodgkin lymphoma. J Nucl Med 60:207-211

25. Zanoni L, Cerci JJ, Fanti S (2011) Use of PET/CT to evaluate response to therapy in lymphoma. Q J Nucl Med Mol Imaging 55:633-647

26. Okada M, Sato N, Ishii K et al (2010) FDG PET/CT versus CT, MR imaging, and 67Ga scintigraphy in the post therapy evaluation of malignant lymphoma. Radiographics 30:939-957

27. Itti E, Lin C, Dupuis J et al (2009) Prognostic value of 18F-FDG PET in patients with diffuse large B-Cell lymphoma: SUV-based assessment at 4 cycles of chemotherapy. J Nucl Med 50:527-533

28. Zhou Y, Zhao Z, Li J et al (2019) Prognostic values of baseline, interim and end-of therapy 18F-FDG PET/CT in patients with follicular lymphoma. Cancer Manage Res 11:6871-6885

29. Kim HJ, Lee R, Choi H et al (2018) Application of quantitative indexes of FDG PET to treatment response evaluation in indolent lymphoma. Nucl Med Mol Imaging 52(5):342-349

\section{Publisher's Note}

Springer Nature remains neutral with regard to jurisdictional claims in published maps and institutional affiliations.

\section{Submit your manuscript to a SpringerOpen ${ }^{\odot}$ journal and benefit from:}

- Convenient online submission

- Rigorous peer review

- Open access: articles freely available online

- High visibility within the field

- Retaining the copyright to your article

Submit your next manuscript at springeropen.com 